\title{
The relation between intelligence and critical flicker fusion
}

JOSEPH R. CAUTELA AND DAVID H. BARLOW

BOSTON COLLEGE

\begin{abstract}
Abstraet
Previous investigations concerning the relationship of Flicker Fusion Threshold (FFT) and intelligence show contradictory results. Using the method of limits, FFT levels were determined on 40 Boston College undergraduates. The FFT scores were negatively correlated with the Otis Quick Scoring Intelligence Tests and College Board tests for Verbal and Mathematical ability. No relationship was found between FFT and class rank. The results are compared with other studies.
\end{abstract}

\section{Introduction}

In recent years there has been an increasing concern in exploring the relation between Flicker Fusion Frequency (FFT) and relevant variables. Of the variables explored, relatively little attention has been given to the variable of intelligence. Not only is the nature of the relation between FFT and Intelligence an important one in its own right, it is also an important parameter when the relation of FFT and other variables is investigated. The importance of control of the intelligence variable has been neglected in the investigation of FFT. Particularly noteworthy in this regard have been studies concerned with the relation of FFT and anxiety (Krugman, 1947; Buhler, 1955; Goldstone, 1955; Jones, 1958; Wagoner, 1960)。These studies indicate an inverse relation between FFT and anxiety。 The studies often use hospital patients as the anxious and non-hospital patients as the non-anxious control group. No attempt was made in any of the studies exploring the relation between FFT and anxiety to control for the intelligence variable. Our own investigation (Cautela \& Barlow, 1965) using college students, showed no relation between anxiety and FFT. It is quite possible that the lack of agreement of our results with previous studies is due to the intelligence factor. If intelligence is inversely related to FFT, then the results of studies of FFT and anxiety cannot be attributed to the anxiety variable alone.

The studies concerning the relation between FFT and intelligence report contradictory results. Colgan (1954) found a positive correlation between FFT and the full scale Wechsler-Bellevue. Tanner (1950) reports slight negative correlations between FFT and various measures of intelligence. Banatl, Clark, \& Lipton (1962) found an insignificant $(p<.05)$ correlation with the full scale Wechsler-Bellevue but a significant ( $p>$.01) negative correlation for the Otis Quick Scoring Intelligence Test. Accordingly, the purpose of this study is to determine the relationship between FFT and intelligence.

\begin{abstract}
Method
Subjects

The Ss wene 40 male Boston College undergraduates. Apparatus
\end{abstract}

The various intelligence measures used were: the Otis Quick Scoring Intelligence Test, Verbal and Mathematical Aptitude Scores on the College Board Entrance Examinations. Class rank was also includeci to investigate the relationship between performance and CFF. The CFF apparatus consisted of a Flicker perception system, model FP-104, including a Sylvania R1131C glow modulator tube placed in an OFC-1 monocular optical system, which avoided the problem of light adaptation. This apparatus is distributed by International Applied Science Laboratory, Inc. A chin rest was also employed.

Procedures

The Otis tests were administered by the University to the students during their freshman year. The College Board scores were obtained from the students records as was the class rank.

The CFF scores were obtained in the following manner: The $S$ entered the room and was asked to be seated by the $\mathrm{E}$. He was then given instructions: "Place your head in the chin rest and look into the tube and you will see a light, sometimes it will be flickering and sometimes it will be steady." The S then adjusted the optical system until he saw the light. "Here it's flickering," at a frequency of $50 \mathrm{cps}$. "Each time you hear the knob click, tell me whether the light is flickering or not. If it's flickering say, yes, if it's not say, no." The $E$ then proceeded to administer a method of limits consisting of 3 blocks of 5 ascending and 5 descending trials in a randomized order, with a 30 sec. rest period after each block. The step interval was $1 \mathrm{cps}$, and the runs were terminated after three successive like responses. Reaction time was taken for each threshold response to the nearest second. Each stimulus, set at a lightdark ratio of $1: 1$, appeared at a brightness of $50 \mathrm{ma}$. The brightness was checked before each $\mathrm{S}$ by a 210 Light Index Meter and the frequency was constantly calibrated by a calibrator included in the FP-104.

\section{Results and Diseussion}

Significant negative Pearson Product Moment correlations were found between FFT and Otis scores $(-.589, \mathrm{p}<.01)$ with an $\mathrm{N}$ of 40 ; between FFT and Verbal Aptitude (VA) on the C.E.E.B. $(-.509, p<.01)$ with an $\mathrm{N}$ of 46 ; and between FFT and Mathematical Aptitude (MA) on the C.E.E.B. $(-.355, \mathrm{p}<.05)$ with an 
$\mathrm{N}$ of 45 . The correlation between FFT and class rank with an $\mathrm{N}$ of 40 was zero. It is interesting to note that rank which is strictly a performance measure is not significant. Also, the two verbal measures (IQ and VA) are much more significant than the mathematical aptitude. The Otis Quick Scoring Intelligence Test is a highly verbal measure of intelligence as is the Verbal Aptitude.

The above results indicate that CFF is more highly related to aptitude measures than to performance measures. Also, verbal aptitude is more significantly related to $\mathrm{CFF}$ than mathematical (or number) aptitude. These data, of course, mean the higher the intelligence, the higher the CFF threshold; for low CFF scores mean higher thresholds.

It is quite difficult to account for the results, either in terms of previous studies or our knowledge of brain physiology. As previously noted, the other studies present contradictory results. An interesting observation in this respect is the fact that the studies that report either a positive correlation (Colgan, 1954) or an insignificant correlation (Banatl, Clark, \& Lipton, 1962) between FFT and intelligence, used the WechslerBellevue as a measure of intelligence. The studies reporting a negative correlation between FFT and intelligence used group intelligence tests as used in this study. Tanner (1950) used the American Council Psychological Examination (ACE), and Banatl, Clark, \& Lipton (1962) used the Otis Quick Scoring Intelligence Test as a measure of intelligence. Perhaps lack of consistency of results concerned with FFT and intelligence is due to some differential factor in the administration of the intelligence tests. The studies reporting a negative correlation used group intelligence tests which were administered in a group situation and required reading on the part of the Ss. The other studies using the Wechsler-Bellevue, of course, employed an intelligence test that was administered individually and did not require reading on the part of the Ss. It is difficult to ascertain from the present study whether these differences do contribute to the lack of consistency of results. A further study is needed in which one group is given the Wechsler-Bellevue and a group intelligence test is administered to the group as a whole. In this study another equated group is also given the WechslerBellevue, and the group intelligence test is administered individually. Correlation with FFT scores would then clarify this point. Of course, there is always the possibility that the difference in results is due to some variable as yet undiscovered.

\section{References}

Banatl, E. S., Clark, M., \& Lipton, J. Critical flicker fusion in relation to Cattells Culture Fair Intelligence Score. Amer. J. Psychol., 1962, 75, 324-325.

Buhler, R. A. Flicker fusion threshold and anxiety level. Dissert. Abstr., 1954, 14, 1255.

Cautela, J. R., \& Barlow, D. The relation between anxiety and flicker fusion threshold in a non-stressful situation. Psychology, $1965,2,8-12$.

Colgan, C. M. Critical flicker fusion, age and intelligence. Amer. J. Psychol., 1954, 67, 711-713.

Goldstone, S. Flisker fusion measurements and anxiety level. I. exp. Psychol., 1955, 49, 200-208.

Jones, O. Relationship between visual and auditory discrimination and anxiety level. J. gen. Psychol., 1958, 59, 111-118.

Tanner, W. P. A preliminary investigation of the relationship between visual fusion of intermittent light and intelligence. Science, 1950, 112, 201-203.

Wagoner, R. A. Differences in response latency and response variability between high and low anxiety subjects in a flicker fusion task. J. abnorm. soc. Psychol., 1960, 61, 355-359.

Wagoner, R., \& Cohen, L. D. Analysis of patterns of response of anxious and non-anxious subjects to a flickering light. Percept. mot. Skills, 1956, 6, 167-170. 